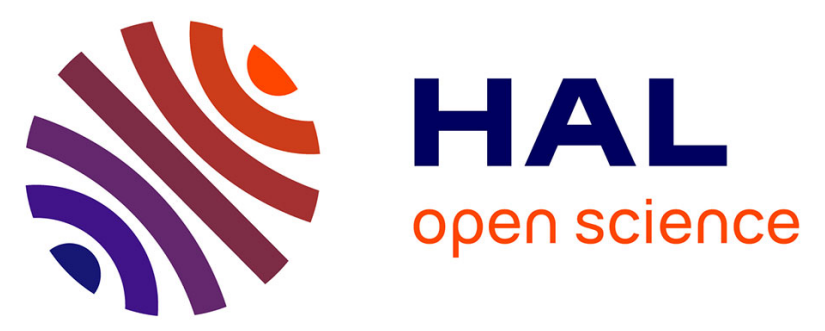

\title{
Non-Invasive Low Cost Method for Linear and Angular Accelerations Measurement in Biped Locomotion Mechanism
}

Viacheslav Khomenko, Patrick Henaff, Olivier Bruneau, Fethi Ben Ouezdou, Vladimir Borysenko, Artem Melnyk

\section{To cite this version:}

Viacheslav Khomenko, Patrick Henaff, Olivier Bruneau, Fethi Ben Ouezdou, Vladimir Borysenko, et al.. Non-Invasive Low Cost Method for Linear and Angular Accelerations Measurement in Biped Locomotion Mechanism. IEEE International Conference SENSORS 2011, Oct 2011, Limerik, Ireland. pp.1756-1759. hal-00667677

\section{HAL Id: hal-00667677 https://hal.science/hal-00667677}

Submitted on 8 Feb 2012

HAL is a multi-disciplinary open access archive for the deposit and dissemination of scientific research documents, whether they are published or not. The documents may come from teaching and research institutions in France or abroad, or from public or private research centers.
L'archive ouverte pluridisciplinaire HAL, est destinée au dépôt et à la diffusion de documents scientifiques de niveau recherche, publiés ou non, émanant des établissements d'enseignement et de recherche français ou étrangers, des laboratoires publics ou privés. 


\title{
Non-Invasive Low Cost Method for Linear and Angular Accelerations Measurement in Biped Locomotion Mechanisms
}

\author{
Viacheslav Khomenko, \\ Olivier Bruneau, Fethi Ben Ouezdou \\ LISV laboratory \\ Versailles St-Quentin-en-Yvelines University \\ 10-12 av. de l'Europe, 78140 Velizy, France \\ slava.khomenko@gmail.com
}

\author{
Patrick Henaff, \\ Artem Melnyk \\ ETIS UMR 8051, UCP-ENSEA-CNRS \\ Cergy-Pontoise University \\ F-95000 Cergy Pontoise, France \\ patrick.henaff@u-cergy.fr
}

\author{
Volodymyr Borysenko \\ Electrotechnical Department \\ Donetsk National Technical University \\ 58 Artem Street, 83001 Donetsk, Ukraine
}

\begin{abstract}
A new non-invasive low cost method for accelerations measurement in biped locomotion mechanisms is presented. The aim is to measure all the joint accelerations of the locomotion system during the walking that is difficult because of the nonaccessibility of several joint axes. Our measurement approach is non-direct using two triaxial accelerometers aligned on the main axis of each body, that ensures measurement of five accelerations per joint, two angular and three linear. By considering negligible the vertical rotation of a leg in the absolute frame during walking, the vertical rotational acceleration of the hip is measurable by two accelerometers aligned along the sagittal axis. For tibia and thigh, the accelerometers are aligned along vertical axes. The calculation of accelerations in articulation references is achieved by solving a system of Newton mechanics equations. The theoretical statements were validated experimentally on the robot for vertical flexion-extension movement.
\end{abstract}

\section{INTRODUCTION}

Non-invasive, or body mounted systems for kinematic analysis of polyarticulated mechanisms are mainly developed since ' 90 s of the XX century, with large proliferation of MEMS sensors [1-5]. Commercialized inertial sensors dedicated to measure displacements and velocities of legged locomotion mechanisms [5] exist, but they are generally expensive and their frequency bandwidth is not enough for acceleration measurement that is important for motion analysis and control. Usually it is a combination of linear accelerometers, of gyroscopes and magnetometers. The signals are treated by Kalman filters [6], but this approach creates integration and derivation errors. Many authors proposed accelerometer-only based approaches [1-3, 7-15].

There are many works concerning measuring of angular accelerations exclusively with linear mono-axis accelerometers that date the second part of the previous century [7, 8]. One of the first [7] is limited only to in-plane movements. In-space measurements were presented in [8], but this method uses disks rotating with constant angular frequency. More recent researches consider measurement systems without rotating parts with five, six and more accelerometers axes placed in orthogonal way on the body [1, 9-15]. Their biggest problems are the singularities in determination of the solutions $[10,11]$. Avoiding singularities is achieved using 9 or 12 axis accelerometer setups, when accelerometers are placed for example along the three axes of a cube, and one in its center. Stability analysis [12] proves that this method gives very good results and can be used for calibration purposes, in biomechanics [13, 14] and robotics [12]. But in practical applications such redundancy is not always needed and less quantity of information is usually acceptable, e.g. the 8 accelerometers setup was used in 6-DOF accelerations measurement in earthquake analysis [15].

However, all these methods require a large number of accelerometers that must be positioned in places of the bodies which are not necessarily accessible without modifying the mechanical conception of the mechanism [5]. Moreover, there are very few works focused on applications for mechanisms with more than one moving body. For polyarticulated systems the number of sensors grows proportionally to the number of 


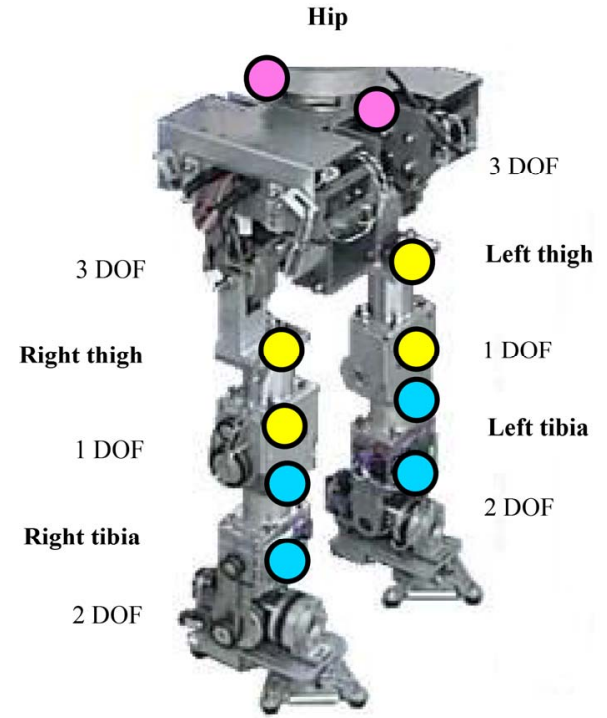

Figure 1. The biped robot. Two aligned accelerometers per body allow five acceleration measurements per articulation.

bodies. That affects the reliability and maintenance costs, thus making the measurement system very expensive and not attractive for practical implementation.

An interesting approach for a locomotion mechanism was proposed in [1]. This method is based on the use of two aligned triaxial accelerometers on the thigh, but the drawback is that the hip rotations are not measured and only linear accelerations of the hip relative to the global coordinate system are taken into account. The models and experimental results are given for different two-segment systems (one human leg and one motorized arm).

Our approach is based on the same technique but extended to the entire biped locomotion system (Fig. 1). We use four accelerometers per leg and two on the hip. Thus we can calculate five accelerations for each articulation included the accelerations of the hip around vertical and frontal axes.

This paper is divided into four sections. After this introduction, the second section presents the methodology of our approach. The mathematical model corresponding to the proposed sensor combination is given and the measurement algorithm is described. The third section gives experimental results for vertical flexion-extension movement of a biped robot. The paper is terminated with conclusions and further work.

\section{Methodology}

\section{A. Mathematical Model of Measurement}

We consider the biped mechanisms hip as the nodal part of a body (Fig. 2). Due to the symmetry of a biped construction in relation to its hip, we present the mathematical model for the hip and one leg (left or right). The inertial frame is denoted as $\{0, x, y, z\}$, three presented local frames are denoted as: $\left\{h_{0}, x_{h}, y_{h}, z_{h}\right\}$ for the hip, $\left\{t_{0}, x_{t h}, y_{t h}, z_{t h}\right\}$ for the thigh and $\left\{t i_{0}, x_{t i}, y_{t i}, z_{t i}\right\}$ for the tibia.

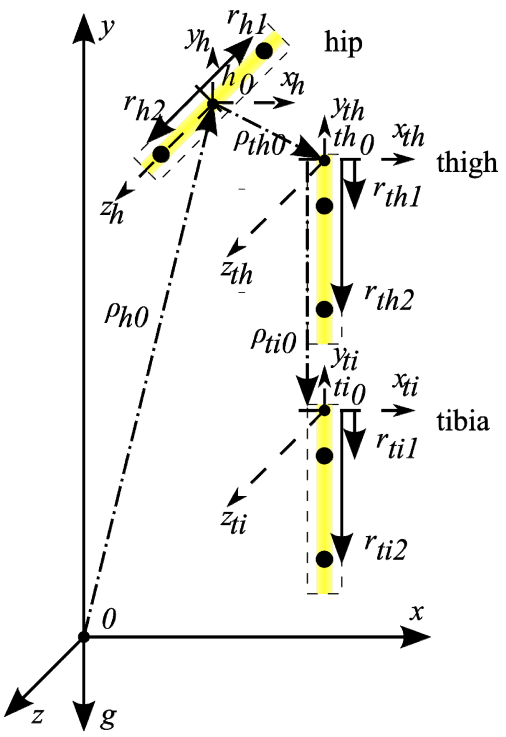

Figure 2. Reference systems. The hip, thigh and tibia. One leg model is presented for more clarity.

The measured hip movements are: 3 linear (along $x, y, z$ axes of the inertial frame) and 2 rotational (which we consider in the local frame of the hip around vertical and frontal axes $\left.y_{h}, z_{h}\right)$. The thigh is connected to the hip; it can rotate around its frontal and sagittal axes $\left(x_{t h}, z_{t h}\right)$. The tibia is connected to the thigh and it has only 1 axis of rotation $\left(z_{t i}\right)$.

The model was constructed in assumption that there is an ideal contact between foot and floor (the rotation of foot relative to the floor is considering negligible) and walking is normal (e.g. stable movement on a surface). The accelerations given by accelerometer sensors positioned on the mechanism as shown on the Fig. 2 were received in general form based on Newton formalism:

$$
\left.\begin{array}{rl}
\overline{\mathbf{a}}_{h 1,2}= & \overline{\boldsymbol{\alpha}}_{h} \times \overline{\mathbf{r}}_{h 1,2}+\overline{\boldsymbol{\omega}}_{h} \times\left(\overline{\boldsymbol{\omega}}_{h} \times \overline{\mathbf{r}}_{h 1,2}\right)+\bar{q}_{h} \cdot\left(\overline{\mathbf{a}}_{h 0}+\overline{\mathbf{g}}\right) \cdot \bar{q}_{h}^{*} ; \\
\overline{\mathbf{a}}_{t h 1,2}= & {\left[\overline{\mathbf{a}}_{h 0}+\overline{\boldsymbol{\alpha}}_{h} \times \overline{\boldsymbol{\rho}}_{t h}+\overline{\boldsymbol{\omega}}_{h} \times\left(\overline{\boldsymbol{\omega}}_{h} \times \overline{\boldsymbol{\rho}}_{t h}\right)\right]+\bar{q}_{t h} \bar{q}_{h} \cdot \overline{\mathbf{g}} \cdot \bar{q}_{h}^{*} \bar{q}_{t h}^{*}+} \\
& +\overline{\boldsymbol{\alpha}}_{t h} \times \overline{\mathbf{r}}_{t h 1,2}+\overline{\boldsymbol{\omega}}_{t h} \times\left(\overline{\boldsymbol{\omega}}_{t h} \times \overline{\mathbf{r}}_{t h 1,2}\right) ; \\
\overline{\mathbf{a}}_{t i 1,2}= & {\left[\overline{\mathbf{a}}_{h 0}+\overline{\boldsymbol{\alpha}}_{h} \times \overline{\boldsymbol{\rho}}_{t h}+\overline{\boldsymbol{\omega}}_{h} \times\left(\overline{\boldsymbol{\omega}}_{h} \times \overline{\boldsymbol{\rho}}_{t h}\right)\right]+} \\
& +\left[\overline{\boldsymbol{\alpha}}_{t h} \times \overline{\boldsymbol{\rho}}_{t i}+\overline{\boldsymbol{\omega}}_{t h} \times\left(\overline{\boldsymbol{\omega}}_{t h} \times \overline{\boldsymbol{\rho}}_{t i}\right)\right]+\bar{q}_{t i} \bar{q}_{t h} \bar{q}_{h} \cdot \overline{\mathbf{g}} \cdot \bar{q}_{h}^{*} \bar{q}_{t h}^{*} \bar{q}_{t i}^{*}+ \\
& +\overline{\boldsymbol{\alpha}}_{t i} \times \overline{\mathbf{r}}_{t i 1,2}+\overline{\boldsymbol{\omega}}_{t i} \times\left(\overline{\boldsymbol{\omega}}_{t i} \times \overline{\mathbf{r}}_{t i 1,2}\right),
\end{array}\right\}
$$

where $\quad \overline{\mathbf{a}}_{h 1,2}, \quad \overline{\mathbf{a}}_{t h 1,2}$ and $\overline{\mathbf{a}}_{t i 1,2}$ are vectors of linear accelerations measured at the $1^{\text {st }}$ and $2^{\text {nd }}$ positions of the hip, thigh and tibia respectively; $\overline{\boldsymbol{\omega}}$ are angular velocities of the bodies in local frames; $\overline{\boldsymbol{\alpha}}$ are angular accelerations of the bodies in local frames; $\overline{\mathbf{a}}_{h 0}$ are linear accelerations of the hip in inertial frame; $\overline{\mathbf{g}}$ is the gravity vector in inertial frame; $\overline{\mathbf{r}}_{1,2}$ are position vectors of accelerometer sensors placed in the 
bodies local frames; $\overline{\boldsymbol{\rho}}_{t h}$ and $\overline{\boldsymbol{\rho}}_{t i}$ are vectors of bodies mutual placement; $\bar{q}$ are quaternions describing bodies rotations.

For simplification, we detail here only the determination of the analytical solution of the first equation of the system (1) corresponding to two triaxial accelerometer sensors mounted on the hip. It was found that in general case its determinant is zero and thus the solution cannot be found. But, in particular case of a biped locomotion system, the rotational acceleration along one axis can supposed zero, and then the unique solution can be achieved because of cross-axis terms elimination in the vector $\overline{\boldsymbol{\alpha}}_{h} \times \overline{\mathbf{r}}_{h 1,2}$. For the hip, the accelerations are:

$$
\begin{aligned}
& \alpha_{h x}=0 ; \\
& \alpha_{h y}=-\left[a_{h 1 z}-a_{h 2 z}-\alpha_{h C z}\right] / r_{h x} ; \\
& \alpha_{h z}=\left[a_{h 1 y}-a_{h 2 y}-\alpha_{h C y}\right] / r_{h x} \text {; } \\
& a_{h 0 x}=a_{h 1 x}-\alpha_{h C x}-\left(a_{h y} \cdot r_{h 1 z}-a_{h z} \cdot r_{h 1 y}\right)-g_{x} ; \\
& a_{h 0 y}=a_{h 1 y}-\alpha_{h C y}-\left(a_{h z} \cdot r_{h 1 x}-a_{h x} \cdot r_{h 1 z}\right)-g_{y} \text {; } \\
& a_{h 0 z}=a_{h 1 z}-\alpha_{h C z}-\left(a_{h x} \cdot r_{h 1 y}-a_{h y} \cdot r_{h 1 x}\right)-g_{z} \text {, }
\end{aligned}
$$

where $\quad r_{h x, y, z}=r_{h x, y, z 2}-r_{h x, y, z 1}$ are projections of distances between sensors on the local frame coordinate axes; $\alpha_{h x, y, z}$ are rotational accelerations of the hip body; $a_{h 0 x, y, z}$ are translational accelerations of the hip body point " $h h_{0}$ ". And the centrifugal terms of accelerations $\alpha_{h C x}, \alpha_{h C y}, \alpha_{h C z}$ are:

$$
\begin{aligned}
& \overline{\mathbf{a}}_{h C}=\overline{\boldsymbol{\omega}}_{h} \times\left(\overline{\boldsymbol{\omega}}_{h} \times \overline{\mathbf{r}}_{h}\right)=\left[\begin{array}{l}
\alpha_{h C x} \\
\alpha_{h C y} \\
\alpha_{h C z}
\end{array}\right]= \\
& =\left[\begin{array}{c}
\omega_{h y} \cdot\left(\omega_{h x} \cdot r_{h y}-\omega_{h y} \cdot r_{h x}\right)-\omega_{h z} \cdot\left(\omega_{h z} \cdot r_{h x}-\omega_{h x} \cdot r_{h z}\right) \\
\omega_{h z} \cdot\left(\omega_{h y} \cdot r_{h z}-\omega_{h z} \cdot r_{h y}\right)-\omega_{h x} \cdot\left(\omega_{h x} \cdot r_{h y}-\omega_{h y} \cdot r_{h x}\right) \\
\omega_{h x} \cdot\left(\omega_{h z} \cdot r_{h x}-\omega_{h x} \cdot r_{h z}\right)-\omega_{h y} \cdot\left(\omega_{h y} \cdot r_{h z}-\omega_{h z} \cdot r_{h y}\right)
\end{array}\right] .
\end{aligned}
$$

As one can see from (2), accomplishing the condition of 2 sensors aligning along sagittal axis of the hip (assuming that $\left.r_{h y}=0, r_{h z}=0\right)$ and by taking that the rotation around its sagittal axis does not an important value $\left(\omega_{h z} \approx 0\right)$ the solution is much simpler:

$$
\left.\begin{array}{l}
\alpha_{h y, z}=\frac{ \pm\left(a_{h 2 z, y}-a_{h 1 z, y}\right)}{r_{h x}} \\
a_{h 0 x, y, z}=\frac{r_{h 1 x} \cdot a_{h 2 x, y, z}-r_{h 2 x} \cdot a_{h 1 x, y, z}}{r_{h x}} .
\end{array}\right\}
$$

The principle showed above is applied for the thigh and tibia, but the bodies' mutual rotations in concordance with (1) should be taken into account. The detailed solutions for them are not presented in this paper because the volume is limited.

\section{B. Algorithm of Measurement}

The algorithm consists of three phases: initialization; calculation of bodies' angular and linear accelerations and quaternion estimation.

The initialization phase is necessary to: 1) parameterize sensors sensitivity coefficients and offsets according to the individual sensors calibration data usually provided by manufacturer; 2) determine mutual orientation of sensors due to possible errors of positioning. Thus, during the initialization phase the biped mechanism should be on the standing posture. The thigh and tibia should be aligned with their main vertical axes, and with the vertical axis of a hip. Equations (1) and (2) should be used on the second phase to determine the bodies' accelerations. The quarternions can be calculated knowing actual orientations of mechanism bodies. It is usually achieved by sensing on the motor level or by estimating angles by the gravity vector in quasi static conditions with accelerometers and then applying a narrow-band low-pass filter.

\section{EXPERIMENTAL RESULTS}

Experimental validation is carried out on the biped anthropomorphic robot of Fig. 1 for vertical flexion-extension movements with frequencies of movements appropriate to the considered mechanism $(0.3-0.7 \mathrm{~Hz})$. All the algorithms are embedded in an industrial electronic real time unit that controls the robot. The principle of sensors alignment allows the differential calculation of accelerations which eliminates the centrifuge terms in the kinematic equations for one body. By combining kinematic equations of two connected bodies one can express five accelerations on the articulation joint axes.

The experimental results are depicted on Fig. 3 for the thigh angular and linear accelerations. For angular accelerations, the results are compared to ones based on usual derivation techniques. It shows that our method produces better sensitivity, less noise and reduced time delay because of larger frequency bandwidth of accelerometers comparing to gyroscopes and inclinometers.

Due to better measurement performances of the accelerometer scheme it is possible to observe parasite oscillations whose origin is in the robot mechanical transmissions elasticities and backlashes. It is necessary to measure these phenomena precisely to decrease their influence on the walk of a robot. The advantage of the accelerometer approach is more evident when the higher resolution is needed (that corresponds to movements with the lowest frequency of $f_{f e}=0.3 \mathrm{~Hz}$ on the Fig. 3). For higher accelerations $\left(f_{f e}=0.7 \mathrm{~Hz}\right)$ the difference between accelerometer and gyroscopic methods in angular accelerations measurements is not so essential. The limiting factor in this case is the narrower bandwidth of a gyroscope. Inclinometer method does not gives good results in both cases. 

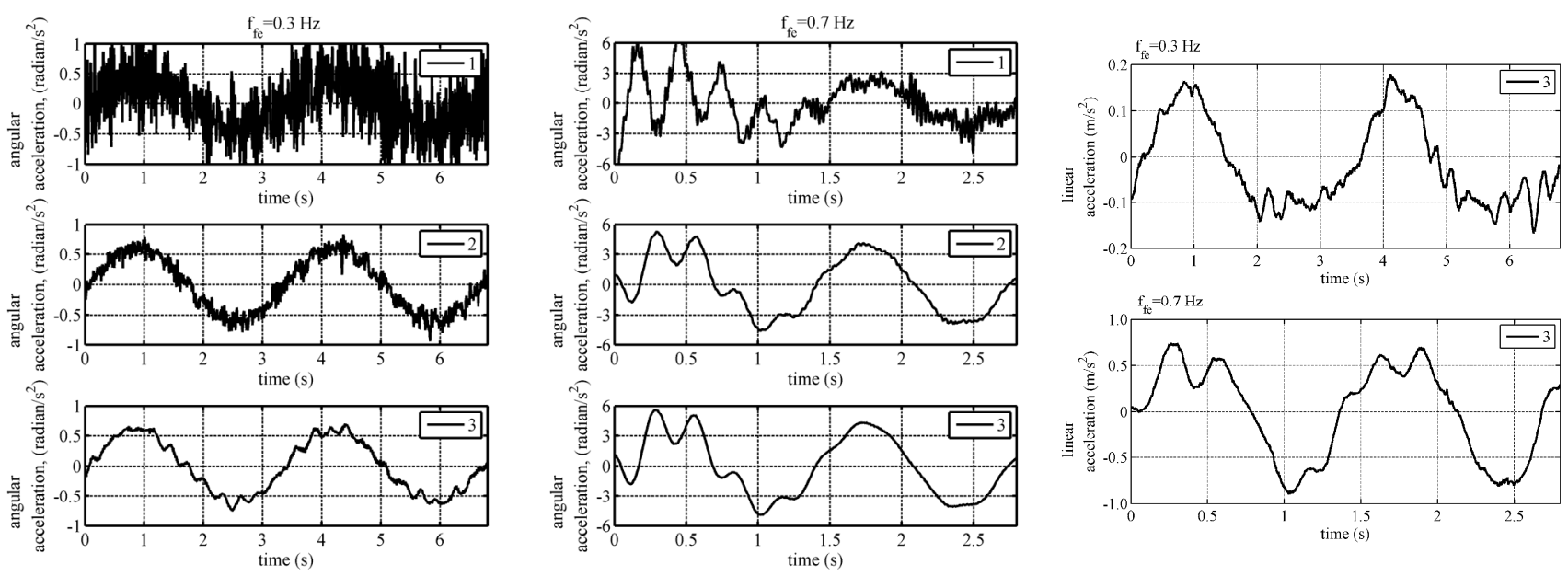

Figure 3. Comparison of three measurement methods for two different frequencies of flexion/extension movement: 1 - double derivation of an inclinometer (SCA102T); 2 - derivation of a gyroscope (LPR530AL); 3- proposed accelerometer setup (CXL04GP3).

\section{CONCLUSION}

A non-invasive method for angular and linear accelerations measurements in poly-articulated mechanisms is proposed. The approach uses the rigid body dynamic theory for a number of triaxial acceleration sensors placed in a specific way on the mechanism. The proposed method was first modeled using Cyberbotics Webots TM software and then validated experimentally on the biped robot ROBIAN, LISV, France, for flexion-extension movements.

The resulting price of the proposed sensor system is about $4000 €$ using ten industrial-grade CXL04GP3 accelerometers. This is a price of one multi-DOF industrial-grade IMU, but at least $1 \mathrm{IMU}$ per mechanisms body is needed. Also, the most of market-presented IMU does not permit to measure body-frame accelerations with acceptable bandwidth. The proposed solution offers observation of main accelerations in a biped locomotion mechanism with sufficient precision, noise level and bandwidth for motion analysis and control. Especially, the method allows to measure accelerations on virtual rotational axes of articulations.

In future, additional analysis of the sensors misalignment on the resulting precision will be made. We are making validation measurements during the walk of a biped robot ROBIAN.

\section{ACKNOWLEDGMENT}

The authors thank to Pr. Y. Alayli, Director of "Laboratoire d'Ingénierie des Systèmes de Versailles, LISV", France, for his useful advices.

\section{REFERENCES}

[1] K. Liu, Y. Inoue, K. Shibata, "Analysis of lower limb segment orientation using triaxial accelerometers," J. of Biomech. Science and Engineering JSME, vol. 5, no. 4, pp. 368-379, 2010.

[2] J. Channells, B. Purcell, R. Barrett, D. James, "Determination of rotational kinematics of the lower leg during sprint running using accelerometers", BioMEMS and Nanotechnology II, Edited by Nicolau, Dan V., Proc. of the SPIE, vol. 6036, pp. 300-308, 2006.

[3] A.T.M.Willemsen, C. Frigo, H.B.K. Boom, "Lower extremity angle measurement with accelerometers - error and sensitivity analysis," IEEE Trans. on Biomedical Engineering 12, pp. 1186-1193, 1991.

[4] R.E. Mayagoitia, A.V. Nene, P.H. Veltink, "Accelerometer and rate gyroscope measurement of kinematics: an inexpansive alternative to optical motion analysis sytems," J. of Biomech. 35, pp. 537-542, 2002.

[5] C.-C. Yang, Y.-L. Hsu, "A review of accelerometry-based wearable motion detectors for physical activity monitoring", Sensors, vol. 10, pp. 7772-7788, 2010.

[6] N. Moriya, P. Harel, I. Moti, "System for three dimensional positioning and tracking. US Patent Publication," US 6691074 B1 International: H03F 1/26, 8 P., published on 10 Febuary 2004.

[7] V.B. Corey, "Measuring Angular Accelerations with Linear Accelerometers," Control Engineering, March 1962.

[8] V. Krishnan, "Measurement of angular velocity and linear acceleration using linear accelerometers", J. of the Franklin Institute, vol. 280, issue 4, pp. 307-315, October 1965.

[9] Z. Qin, L. Baron, L. Birglen, "Robust design of inertial measurement units based on accelerometers", J. Dyn. Sys., Meas., Control, vol. 131, issue 3, 7 P., 2009.

[10] B. Zappa, G. Legnani, A.J. van den Bogert, R. Adamini, "On the number and placement of accelerometers for angular velocity and acceleration determination," J. of Dynamic Systems, Measurement, and Control, vol. 123/552, 2001.

[11] P. Cardou, J. Angeles, "Singularity analysis of accelerometer strapdowns for the estimation of the acceleration field of a planar rigidbody motion", 12th IFToMM World Congress, Besançon, 2007.

[12] P. Cardou, "Computing the Rigid-Body Acceleration Field from Nine Accelerometer Measurements," Advances in Soft Computing, vol. 83, pp. 325-339, 2010.

[13] N. Yoganandan, J. Zhang, F.A. Pintar, Y. King Liu, "Lightweight lowprofile nine-accelerometer package to obtain head angular accelerations in short-duration impacts," J. of Biomechanics, vol. 39, issue 7, pp. 1347-1354, 2006,

[14] D.T.-P. Fong, Y.-Y. Chan, "The use of wearable inertial motion sensors in human lower limb biomechanics studies: a systematic review," Sensors, 10(12), pp. 11556-11565, 2010.

[15] Z. Yang, Y. Shen, Z. Liu, "Measurement of six degree-of-freedom ground motion by using eight accelerometers", Earthquake Engineering and Engineering Vibration, vol. 4, n. 2, pp. 229-232, 2005. 